\title{
E्己̌reAáo \\ Alfabetizacion Estadistica en Profesores de Distintos Niveles Formativos
}

\author{
Francisco Enrique Rodriguez-Alveal \\ 'Universidad del Bío-Bío (UBB), Chillán - Chile
}

RESUMEN - Alfabetizacion Estadistica en Profesores de Distintos Niveles Formativos. El presente artículo tiene por objetivo evaluar los niveles de alfabetización estadística y la percepción que tienen estudiantes en Formación Inicial Docente y profesores en ejercicio del sistema escolar. Para tal efecto, se aplicaron dos instrumentos: el primero, de alfabetización estadística y el segundo de percepción, validados estadística. Los resultados evidencian que, en general, los estudiantes en Formación Inicial Docente y profesores en ejercicio presentan porcentajes de logro descendidos en la descodificación de situaciones problemáticas de tipo textual, entregando además argumentaciones poco plausibles con los saberes estadísticos donde intervienen conceptos básicos de estadística.

Palabras-clave: Alfabetización Estadística. Formación Inicial Docente. Evaluación.

ABSTRACT - Statistical Literacy in Teachers from Distinct Training Levels. The aim of this paper is to assess the levels of statistical literacy and perception of Early Teaching Training (ITE) students and teachers working in the Chilean schooling system. Two measuring tools were applied: (1) statistical literacy and (2) perception, both validated statistically. The results of this analysis show that, in general, ITE students and working teachers present low achievement percentage on decoding textual situations, delivering unlikely arguments with little basis on basic statistical concepts. Keywords: Statistical Literacy. Early Teacher Training. Evaluation.

Educação \& Realidade, Porto Alegre, v. 42, n. 4, p. 1459-1477, oct./dic. 2017. 
Alfabetizacion Estadistica en Profesores de Distintos Niveles Formativos

\section{Introduccion}

Desde el siglo pasado estamos en presencia de una de las revoluciones tecnológicas más extraordinarias de la historia, centrada en las tecnologías de la información y la comunicación tal vez la más importante desde la revolución industrial debido a que ella incide en el conjunto de la actividad humana (Castells, 1999).

Producto de lo anterior hoy en día las personas diariamente se ven en la necesidad de saber interpretar y comprender gran cantidad de información sobre diferentes temas presentes en los medios y deben tomar decisiones que involucran apropiarse de conceptos estadísticos. Es por ello que la incorporación de la estadística en el currículo escolar es una realidad evidente a partir de la enseñanza básica, para formar ciudadanos competentes en la era de la Web 2.0 (Organización para la Cooperación y el Desarrollo Económicos (OCDE), 2011, citado por Serradó 2013). Producto de lo anterior se busca desarrollar en los ciudadanos habilidades tendientes al logro de una alfabetización estadística (Gal, 2004) coherente con las demandas de la actual sociedad.

En este contexto la formación de los escolares en este ámbito juega un rol fundamental debido a la gran cantidad de información económica, educativa, social, cultural y política que se presenta mediante representaciones gráficas o indicadores numéricos. Ejemplo de ello son las cifras que hacen referencia a promedios y porcentajes, como así también los datos en coherencia con el tipo de muestreo cuya finalidad es reforzar la credibilidad de la información resumida. En consecuencia el uso de habilidades de alfabetización estadística se transforma en un conocimiento de utilidad para descodificar y codificar información permitiendo que los usuarios puedan tomar decisiones al respecto luego de develar el o los mensajes implícitos en los resúmenes de los datos.

En este sentido, en los últimos años en Chile se ha fortalecido el currículo escolar con la introducción del eje de Datos y Probabilidad en el sector de matemática (Chile, 2012) en el cual se explicitan contenidos y habilidades (identificar, inferir y reorganizar información) vinculadas a los aprendizajes de los estudiantes, de manera tal que junto con comprender conceptos logren razonar matemáticamente al momento de enfrentar problemas estadísticos. Por otro lado se han hecho explícitos estándares orientadores para egresados de carreras de pedagogía, enfatizando en su formación saberes pedagógicos y disciplinarios (Chile, 2012). Entonces, el profesor debe ser capaz de diseñar actividades y unidades que le permitan conducir el aprendizaje de sus alumnos y alumnas para el desarrollo de habilidades de recolección, organización, interpretación, representación y análisis de datos en contextos variados.

En coherencia con lo anterior se espera que la enseñanza de la estadística no se limite solamente a la memorización de los contenidos entregados por los profesores del sistema escolar, como así también a la resolución mecánica de ejercicios que en la mayoría de las actividades propuestas aluden a situaciones no empíricas, de manera similar la construcción de gráficos. Por el contrario las orientaciones ministeria-

1460 Educação \& Realidade, Porto Alegre, v. 42, n. 4, p. 1459-1477, oct./dic. 2017. 
les dicen relación con comprender, describir y modelar fenómenos de la vida diaria en diversos contextos, por ejemplo entender la variación del Îndice de Precios al Consumidor (IPC), la fluctuación de las tasas de intereses en los bancos, el rendimiento académico entre otros. Es decir, hacer hablar los datos en contextos definidos o interpretar la información estadística resumida en la prensa nacional o internacional como así también ser capaz de criticar los indicadores estadísticos entregados. En otras palabras, como sostiene Mooney (2002), se debe aprender una racionalidad estadística que permita a los individuos descodificar, describir, analizar, codificar, interpretar o criticar la información representada ya sea gráficamente o numéricamente.

Sin embargo, evaluaciones estandarizadas tales como la prueba Trends in International Mathematics and Science Study (TIMSS) (Chile, 2011), muestran logros descendidos de los escolares chilenos en relación al ranking a nivel internacional. Por otro lado estudios a nivel nacional han evidenciado que profesores y profesoras del sistema escolar confunden los objetivos de la enseñanza de la estadística, identificándolos con la introducción de procedimientos algorítmicos en el cálculo de medidas numéricas y en la aplicación de técnicas de resumen de información por medio de tablas y gráficos (Pino; Estrella, 2012; Alveal; Rubilar, 2012). Este tipo de hallazgos son recientes en el país y entregan señales sobre las habilidades de alfabetización estadística que se promueven en los establecimientos escolares. De lo anterior se podrían conjeturar hipótesis sobre los aprendizajes alcanzados por los estudiantes.

En este contexto el propósito del presente estudio es Evaluar los niveles de alfabetización para el aprendizaje de la estadística y la percepción que tienen los estudiantes de Formación Inicial Docente y profesores en ejercicio del sistema escolar, para dar respuesta a los estándares fijados por el Ministerio de Educación Chileno. Para el logro de este propósito los objetivos específicos son:

1. Relacionar los componentes pedagógicos percibidos por los encuestados en relación a las actitudes que tienen hacia la estadística;

2. Comparar las habilidades de alfabetización estadística de los estudiantes en formación inicial docente y profesores en ejercicio del sistema escolar con los estándares fijados por el Ministerio de Educación chileno.

Producto de lo anterior, el presente artículo pretende explorar la relación del componente disposicional y el de conocimientos introducidos en el modelo de Gal (2004) en relación a la alfabetización estadística desde la mirada de un nuevo instrumento, en estudiantes en formación inicial docente y en profesores en ejercicio del sistema escolar.

\section{Discusion Teorica}

La United Nations Educational, Scientific and Cultural Organization (UNESCO) declara que la alfabetización es un derecho humano 
fundamental y constituye la base del aprendizaje a lo largo de toda la vida; por tanto se promueve una alfabetización holística y sostenible dada la capacidad de transformar la vida de las personas (United Nations Educational, Scientific and Cultural Organization, 2015).

Esto quiere decir que el termino alfabetización, tradicionalmente asociado a la lectura y escritura en la lengua materna ha sufrido modificaciones incorporando el cálculo y las habilidades de razonamiento matemático, para dar respuesta a la diversidad de mensajes que circulan en la actualidad en soportes impresos y digitales. Es así como hoy se habla de diferentes tipos: alfabetización científica, alfabetización matemática y alfabetización estadística, entre otros.

En particular, a nivel internacional existen diferentes posturas sobre lo que se entiende por alfabetización estadística. Wallman (1993) la define como la habilidad para entender y evaluar críticamente los resultados estadísticos con los cuales interactuamos en la cotidianeidad. Por su parte Watson (1997) propone un marco que presenta tres niveles: comprensión básica de la terminología estadística y probabilística, comprensión del leguaje estadístico y actitud crítica frente a los resúmenes estadísticos.

Para Garfield, delMas y Chance (2003), la alfabetización estadística involucra habilidades básicas para comprender información estadística y la capacidad de organizar la información y resumirla mediante tablas y gráficos que permitan visualizar el comportamiento de los datos. En particular hace referencia a la capacidad de comprender conceptos básicos, asociados a terminología y símbolos estadísticos de uso común.

Por otro lado Gal (2004) propone un modelo que involucra dos componentes que interactúan de manera conjunta en el proceso de alfabetización estadística. El primero de ellos se relaciona con los conocimientos y está compuesto por cinco elementos cognoscitivos: habilidades de alfabetización, conocimiento estadístico, conocimiento matemático, conocimiento de contexto y habilidades críticas. El segundo componente es de carácter disposicional e involucra una postura crítica, creencias y actitudes.

En coherencia con lo propuesto por la UNESCO en relación a una alfabetización más holística y relacionada con los lineamientos del Ministerio de Educación de Chile (MINEDUC), la propuesta de Gal (2004) resulta de interés abordar a nivel de profesores del sistema escolar chileno, debido a que considera un componente de tipo actitudinal dentro de la alfabetización estadística. En otras palabras integra una suma de emociones y sentimientos que experimentan los alumnos durante el periodo de aprendizaje de la estadística (Gal; Ginsburg, 1997) lo que coincide con una visión pedagógica centrada en el aprendizaje y no de nivel tecnócrata, donde la disposición hacia el saber es un factor a considerar en todo proceso de enseñanza aprendizaje para que este sea efectivo.

Debido que actualmente hay un creciente interés frente a la problemática de la formación de profesores, en particular a nivel de ense- 
ñanza básica respecto a la estadística debido a que es un área menos abordada, a pesar de su reconocida utilidad en la formación ciudadana como así también figurar en las directrices curriculares del Ministerio de Educación chileno.

En este sentido Serradó (2013) hace un análisis de la evolución histórica de la noción de alfabetización a contar de 1951 en adelante, identificando conceptos tales como alfabetización numérica, estadística y cultura estadística términos que permiten interactuar de forma efectiva en la actual sociedad, llamada de la información. A su vez, describe las acciones llevadas a cabo por los miembros del proyecto internacional de alfabetización estadística para el profesorado aportándoles: conocimientos teóricos, recursos para la enseñanza y actividades para promocionar está competencia entre el alumnado, haciendo notar que existen páginas Web que entregan información contextualizada, la cual puede ser utilizada por los profesores en ejercicio, para que los alumnos interactúen con datos reales.

Por otro lado Mafokozi (2011) analiza aspectos relacionados con el proceso enseñanza-aprendizaje de las matemáticas y más específicamente con la estadística evidenciando que los alumnos ingresan a las aulas universitarias españolas con un nivel de alfabetización estadística de cuarto de Educación Secundaria Obligatoria (ESO), equivalente al segundo año de enseñanza media del sistema escolar chileno. Éste es un nivel que no permite predecir de modo fiable el rendimiento en la universidad. Una explicación a esta situación sería la escasa importancia que se otorga a la estadística en el bagaje académico del futuro universitario, como sugiere Roca (2002).

Kataoka et al. (2010) introducen la idea de cultura estadística, mediante actividades contextualizadas e interdisciplinarias a nivel de alumnos de enseñanza básica haciendo uso de ambientes virtuales interactivos de fuente abierta denominado OVALE proyecto a cargo de la Universidad Estadual de Santa Cruz que posee una planilla electrónica de ingreso de información y rutinas de resumen de datos estadísticos de manera gráfica y numérica. Los resultados evidencian que en general los alumnos no son ajenos a los entornos virtuales, como planillas de cálculo y navegación por internet, como así también resulta de interés para ellos interactuar con datos reales producto de situaciones contextualizadas las cuales permite colocar en juego conceptos estadísticos. Cabe mencionar que dicho constructo para algunos autores es denominado alfabetización estadística (Batanero, 2002)

De manera similar Coutinho, Almouloud e Silva (2012) realizaron un estudio en profesores de matemática con el objetivo de construir e interpretar representaciones gráficas haciendo uso del software Geogebra de fuente abierta disponible en internet. Entre los hallazgos se destaca que los profesores objeto de estudio presentaban un adecuado desempeño en lo relacionado con uso de software como así también en la elaboración de representaciones gráficas de manera autónoma. No obstante se evidencian habilidades descendidas en la interpretación de la información resumida en dichas representaciones, es decir, en gene- 
ral los profesores bajo estudio presentan habilidades procedimentales, hallazgos similares a los encontrados por Alveal y Rubilar (2012). Además las actividades permitieron que los profesores generaran un manual tutorial para potenciar de manera didáctica pedagógica el uso del programa Geogebra como una herramienta de resumen de información estadística. Al respecto cabe mencionar que el uso del software Geogebra es promovido en actividades de trabajo autónomo en los textos escolares del sistema escolar chileno.

En coherencia con lo anterior, a nivel nacional, Estrella, Olfos y Mena-Lorca (2015) en un estudio sobre el saber del profesor de enseñanza básica en su conocimiento disciplinario de estadística, entregan evidencias de los bajos desempeños de profesores y sus alumnos en la lectura, interpretación, construcción de tablas de distribución. No obstante estudios tendientes a conocer el nivel de alfabetización de profesores en ejercicio y en formación en el sistema escolar chileno son escasos, en particular Alveal, Fuentes y Rubilar (2016) desarrollan un estudio cuantitativo tendiente a conocer el nivel de alfabetización numérica que poseen estudiantes de Pedagogía Media en Matemática. Los hallazgos entregan resultados descendidos en la habilidad para descodificar información presente en representaciones gráficas y solamente son capaces de realizar cálculos numéricos sin profundizar en la teoría que subyace en las propiedades de los estadígrafos de tendencia central estudiados. Como así también no presentan habilidades para discriminar entre la aplicación de un promedio simple o un promedio ponderado en presencia de situaciones problemáticas.

Por otro lado, Tauber, Albrecht y Bertorello (2011) concluyen que es de interés investigar sobre las actitudes y formas de pensamiento de los futuros profesores en torno a los conceptos de estadística. A su vez Cravero, Redondo y Santellán (2011) comentan que no existe un instrumento que permita determinar de manera especifica si un individuo y en particular los profesores en ejercicio y estudiantes en formación inicial docente están estadísticamente alfabetizados.

Además del componente cognoscitivo, otro factor que juega un rol fundamental en la entrega de contenidos es la actitud disposicional que presentan los estudiantes en formación inicial docente en el aula, situación que conlleva conocer cómo se sienten y valoran los contenidos entregados por los docentes, los cuales deberán reproducir en el mundo laboral acorde a los requerimientos del MINEDUC, plasmados en los estándares disciplinarios.

\section{Método y Tecnicas}

\section{Diseño del Estudio}

Investigación de diseño cuantitativo de tipo descriptivo, comparativo y analítico que permite indagar el comportamiento y la existencia de diferencias significativas entre los dos grupos bajo estudio. Además se utilizó complementariedad metodológica mediante técnicas cualita- 
tivas para analizar las narrativas de los estudiantes en formación inicial docente y profesores en servicio. Los fragmentos de texto de unidad variable configuran ideas básicas a través de las cuales se apoyan y articulan sus experiencias (Nieto; Barrantes, 2003). En resumen mediante este paradigma mixto se pretende comparar las habilidades asociadas a la alfabetización estadística, presentes en profesores en ejercicio del sistema escolar y estudiantes en Formación Inicial Docente de una universidad del centro sur del país.

\section{Participantes}

Para efectos del estudio se realizó un muestreo probabilístico del tipo estratificado considerando una afijación proporcional al tamaño de los estratos, con una varianza máxima y un error de un 5\% (Azorín; Sánchez, 1986). El tamaño de muestra mínimo es de 103 sujetos, de los cuales 88 corresponden a estudiantes en Formación Inicial Docente (FID) de una universidad del centro sur del país de carácter estatal y pública. Específicamente el 76,6 \% de los estudiantes de esta institución pertenecen a los tres primeros quintiles y el $42,1 \%$ de sus estudiantes proviene de establecimientos municipales, 54,7 \% de establecimientos particulares subvencionados y solamente un 3,2 \% de establecimientos particulares pagados. Por su parte el segundo estrato de la muestra está compuesto por 15 profesores en ejercicio del sistema escolar que asisten a un curso de perfeccionamiento en la misma institución.

En la siguiente tabla (Tabla 1) se muestra la caracterización de los sujetos encuestados, se puede observar que no existe diferencias estadísticamente significativas en la distribución de género (hombres y mujeres). Por otro lado, los profesores del sistema escolar tienen una edad promedio de 32,3 años y los estudiantes en Formación Inicial Docente de 23,2 años. Cabe mencionar que los profesores presentan en promedio 7,5 años de servicio con una distribución relativamente heterogénea (D.E=8,0 años) y asimétrica positiva (C.A=0,7), desempeñándose mayoritariamente en el sistema municipal ${ }^{1}(60,0 \%)$. Por su parte los estudiantes FID encuestados provienen en general del sistema particular subvencionado (65,0\%), no obstante el 34,0\% realizó estudios en el sistema municipal (p-valor=0,05). 
Alfabetizacion Estadistica en Profesores de Distintos Niveles Formativos

Tabla 1 - Estadísticas Básicas de los Estudiantes en Formación Inicial Docente y Profesores del Sistema Escolar Considerados en el Estudio

\begin{tabular}{|r|c|c|c|}
\hline & $\begin{array}{c}\text { Estudiantes en } \\
\text { Formación Inicial } \\
\text { Docente }\end{array}$ & $\begin{array}{c}\text { Profesores del } \\
\text { Sistema Escolar }\end{array}$ & p-Valor \\
\hline Fenero & & & \\
\hline Masculino & $31,8 \%$ & $60,0 \%$ & \multirow{2}{*}{0,51} \\
\hline Edad (años) & $23,2(4,9)$ & $40,0 \%$ & 0,00 \\
\hline Años de Servicio & --- & $32,3(11,0)$ & \\
\hline Dependencia & & $7,5(8,0)$ & \multirow{2}{*}{0,05} \\
\hline Municipal & 34,0 & 60,0 & \\
\hline P. Subvencionado & 66,0 & 40,0 & \\
\hline
\end{tabular}

Fuente: Elaboración propia.

\section{Instrumentos de Recoleccion de Datos}

Para efectos del estudio se aplicaron dos instrumentos: Uno sobre alfabetización estadística y otro sobre percepción de actitudes hacia la estadística. El primero fue elaborado por el investigador en coherencia con el modelo propuesto por Gal (2004) y consistió en una prueba del tipo lápiz y papel de selección múltiple. El análisis de fiabilidad realizado mediante el coeficiente Alfa Cronbach arrojó un valor de $\alpha=0,65$, indicando que posee una consistencia interna adecuada para los efectos del estudio. En la siguiente tabla (Tabla 2) se muestran las especificaciones del instrumento.

Tabla 2 - Tabla de Especificaciones del Instrumento de Conocimientos Relacionados con la Alfabetización Estadística según Modelo de Gal (2004)

\begin{tabular}{|l|l|c|}
\hline Contenido & Habilidad & Nro. Preg. \\
\hline $\begin{array}{l}\text { Conceptos básicos de muestreo } \\
\text { estadístico }\end{array}$ & $\begin{array}{l}\text { Comprender la diferencia entre } \\
\text { población de interés y población } \\
\text { de donde se extrae la muestra y } \\
\text { representatividad }\end{array}$ & 2 \\
\hline $\begin{array}{l}\text { Tablas de distribución de frecuen- } \\
\text { cias porcentuales de doble entrada } \\
\text { y representaciones gráficas bidi- } \\
\text { mensionales }\end{array}$ & $\begin{array}{l}\text { Comprender el significado de los } \\
\text { porcentajes y frecuencias obser- } \\
\text { vadas en información unidimen- } \\
\text { sional y bidimensional }\end{array}$ & 2 \\
\hline Resúmenes estadísticos numéricos & $\begin{array}{l}\text { Interpretar lo que significa un es- } \\
\text { tadístico y/o parámetro y hacerse } \\
\text { preguntas sobre él: iPuede ser posi- } \\
\text { ble?, iEs representativo?, ¿Se utiliza } \\
\text { para describir o para generalizar? }\end{array}$ & 2 \\
\hline
\end{tabular}

Fuente: Elaboración propia.

1466 Educação \& Realidade, Porto Alegre, v. 42, n. 4, p. 1459-1477, oct./dic. 2017. 
El segundo instrumento aplicado fue la Escala de Actitudes hacia la Estadística introducida por Estrada (2011) del tipo likert con cinco opciones de respuesta (Muy de acuerdo, de acuerdo, indiferente, en desacuerdo, muy en desacuerdo). Para efectos del estudio se consideraran solamente los reactivos del componente pedagógico cognitivo y los del componente antropológico (social, educativa e instrumental). En la siguiente tabla (Tabla 3) se muestran los aspectos evaluados.

Tabla 3 - Tabla de Especificaciones del Instrumento Escala de Actitudes hacia la Estadística de Estrada (EAEE) para Profesores en Formación y en Ejercicio

\begin{tabular}{|l|c|c|c|}
\hline & \multicolumn{3}{|c|}{ Componentes Pedagógicos } \\
\hline Componente Antropológico & Afectivo & Cognitivo & Comportamental \\
\hline Educativa & $7-12-23$ & $4-6-17$ & $8-15-22$ \\
\hline
\end{tabular}

Fuente: Elaboración propia.

La fiabilidad el instrumento fue medida mediante el coeficiente Alpha de Cronbach $\alpha=0,84$ (IC 95\% $(0,77-0,88)$ ) lo cual indica que el instrumento posee una buena consistencia interna. Además se estudió la validez del constructor del instrumento mediante un análisis factorial de componentes principales mediante la rotación varimax. El índice de adecuación muestral de KMO fue de 0,79 cuyo valor es considerado adecuado, así como la prueba de esfericidad de Bartlett, $\chi^{2}=281,4 ; p<0,00$. Lo cual nos permiten inferir que el instrumento reúne las propiedades psicométricas adecuadas, para efectos del estudio.

\section{Procedimiento de Análisis}

Los resultados se presentan mediante un análisis descriptivo numérico (porcentaje, promedio, desviación estándar, coeficiente de asimetría y de correlación) y gráfico mediante representaciones que permiten visualizar el comportamiento distribucional de las respuestas de los encuestados. Complementariamente se utilizó la prueba de comparación de proporciones para verificar la existencia de diferencias significativas en los porcentajes de logro promedio en las respuestas. A se consideró un análisis factorial de la varianza a una o dos vías considerando un nivel de significación del $5 \%(a=0,05)$. El procesamiento de la información cuantitativa fue realizado en el paquete estadístico SPSS 13.0 y la información cualitativa en el software Qualitative Data Analysis (QDA).

\section{Resultados}

Los resultados se presentan en dos secciones, en coherencia con los objetivos del estudio. En primer lugar, se entregan los resultados promedios acerca de la actitud de los encuestados hacia la estadística derivados de la aplicación del instrumento introducido por Estrada. En segundo lugar, se presentan los porcentajes de logro en relación a las 
Alfabetizacion Estadistica en Profesores de Distintos Niveles Formativos

habilidades de alfabetización estadística de los alumnos en Formación Inicial Docente (FID) y profesores en ejercicio del sistema escolar. Se discuten los argumentos que esgrimen los sujetos, en relación a la descodificación de las situaciones problemáticas entregadas en las cuales se han puesto en juego conceptos estadísticos de uso común.

\section{Actitudes hacia la Estadística}

En la Tabla 4 se muestran las estadísticas básicas de los componentes pedagógicos (afectivo, cognitivo y comportamental) de los estudiantes en FID y profesores en ejercicio del sistema escolar dentro del componente antropológico. En general, se observa que en promedio no existen diferencias significativas entre los grupos bajo estudio, salvo en el componente afectivo que dice relación con la afirmación encuentro interesante el mundo de la estadística, donde los estudiantes en FID presentan un resultado promedio más descendido $(3,7)$ que los profesores en ejercicio del sistema escolar $(4,5)$, siendo estos últimos un grupo de carácter más heterogéneo en su respuesta. Lo anterior puede ser explicado debido a que los profesores en su práctica docente actualmente pueden hacer uso de material educativo donde se introducen conceptos estadísticos para resolver situaciones del diario vivir; en cambio los estudiantes en FID tienen una formación de carácter algorítmica (Estrella; Olfos; Mena-Lorca, 2015).

Tabla 4 - Estadísticas Básicas de los Componentes Pedagógicos según Componente Antropológico Educativo, según Grupo de Estudio

\begin{tabular}{|c|c|c|c|}
\hline Componentes Pedagógicos & $\begin{array}{c}\text { Estudiantes en } \\
\text { Formación Inicial } \\
\text { Docente }\end{array}$ & $\begin{array}{c}\text { Profesores en } \\
\text { Ejercicio sistema } \\
\text { Escolar } \\
\end{array}$ & Valor $\mathrm{p}$ \\
\hline \multicolumn{4}{|l|}{ Afectivo } \\
\hline $\begin{array}{r}\text { Me divierto en las clases que se explica } \\
\text { estadística }\end{array}$ & $3,2 \pm 0,9_{\mathrm{a}}$ & $3,8 \pm 0,41_{\mathrm{a}}$ & 0,10 \\
\hline $\begin{array}{r}\text { Encuentro interesante el mundo de la } \\
\text { estadística }\end{array}$ & $3,7 \pm 0,7_{\mathrm{a}}$ & $4,5 \pm 0,55_{\mathrm{b}}$ & 0,04 \\
\hline $\begin{array}{r}\text { Si pudiera eliminar alguna materia } \\
\text { sería la estadística }\end{array}$ & $4,5 \pm 0,8_{\mathrm{a}}$ & $4,8 \pm 0,41_{\mathrm{a}}$ & 0,70 \\
\hline \multicolumn{4}{|l|}{ Cognitivo } \\
\hline $\begin{array}{r}\text { Es fundamental la formación básica } \\
\text { del futuro ciudadano }\end{array}$ & $3,9 \pm 0,7_{\mathrm{a}}$ & $3,8 \pm 1,2_{\mathrm{a}}$ & 1,00 \\
\hline $\begin{array}{r}\begin{array}{r}\text { En la escuela no se habría de enseñar } \\
\text { estadística }\end{array} \\
\end{array}$ & $4,6 \pm 0,7_{\mathrm{a}}$ & $4,5 \pm 0,6 a_{\mathrm{a}}$ & 1,00 \\
\hline La estadística es fácil & $2,9 \pm 0,9 a$ & $3,3 \pm 1,0_{a}$ & 0,67 \\
\hline \multicolumn{4}{|l|}{ Comportamental } \\
\hline $\begin{array}{r}\text { Los problemas de estadística me resul- } \\
\text { tan fáciles }\end{array}$ & $3,1 \pm 0,9_{a}$ & $3,9 \pm 0,4_{\mathrm{b}}$ & 0,00 \\
\hline $\begin{array}{r}\text { En clase de estadística nunca entiendo } \\
\text { de qué están hablando }\end{array}$ & $3,8 \pm 0,8_{a}$ & $4,7 \pm 0,5_{\mathrm{b}}$ & 0,00 \\
\hline $\begin{array}{r}\text { A menudo explico a mis compañeros } \\
\text { problemas de estadística que no han } \\
\text { entendido }\end{array}$ & $2,9 \pm 0,9_{\mathrm{a}}$ & $3,0 \pm 0,8_{\mathrm{a}}$ & 0,31 \\
\hline
\end{tabular}

*Letras iguales indican que no existen diferencias significativas al 5\%. Fuente: Elaboración propia. 
Por otro lado, solamente se observan diferencias significativas al 5\% en el componente comportamental en lo que dice relación a los problemas estadísticos me resultan fáciles y en clase de estadística nunca entiendo de qué están hablando, evidenciándose que los profesores del sistema escolar obtienen un valor promedio más alto.

Para estudiar la relación entre factor grupo (Estudiantes en FID y profesores en ejercicio del sistema escolar), género (Femenino y Masculino) y la interacción entre ambos, se realizó un análisis de la varianza factorial de la puntuación total verificándose los supuestos para el contraste paramétrico. El cual arrojo que solamente el factor grupo tiene un efecto estadísticamente significativo sobre la puntuación media en las escalas de actitudes (valor $\mathrm{p}=0,004$ ), en cambio género (valor $\mathrm{p}=0,33$ ) y la interacción entre ambos (valor $\mathrm{p}=0,89$ ) no presentan diferencias significativas.

Respecto de la diferencia entre grupos, se observa (Tabla 5) una mayor actitud promedio en los profesores en ejercicio del sistema escolar a la que presentan los estudiantes en formación inicial docente la cual es estadísticamente significativa (valor $\mathrm{p}=0,002$ ), la variabilidad en ambos grupos no se presentan diferencias significativas (valor $\mathrm{p}=0,49$ ).

\section{Tabla 5 - Estadísticas del Promedio de la Puntuación Total en Estudiantes en Formación Inicial Docente y Profesores del Sistema Escolar}

\begin{tabular}{|c|c|c|c|}
\hline & $\begin{array}{c}\text { Estudiantes en } \\
\text { Formación Inicial } \\
\text { Docente }\end{array}$ & $\begin{array}{c}\text { Profesores del } \\
\text { Sistema Escolar }\end{array}$ & p-Valor \\
\hline Puntuación Promedio & $3,5 \pm 0,53_{\mathrm{a}}$ & $4,0 \pm 0,41_{\mathrm{b}}$ & 0,002 \\
\hline
\end{tabular}

Fuente: Elaboración propia.

En la siguiente representación gráfica (Figura 1) ilustra el comportamiento distribucional entre los resultados promedios en el grupo de estudiantes en FID y profesores en ejercicio del sistema escolar.

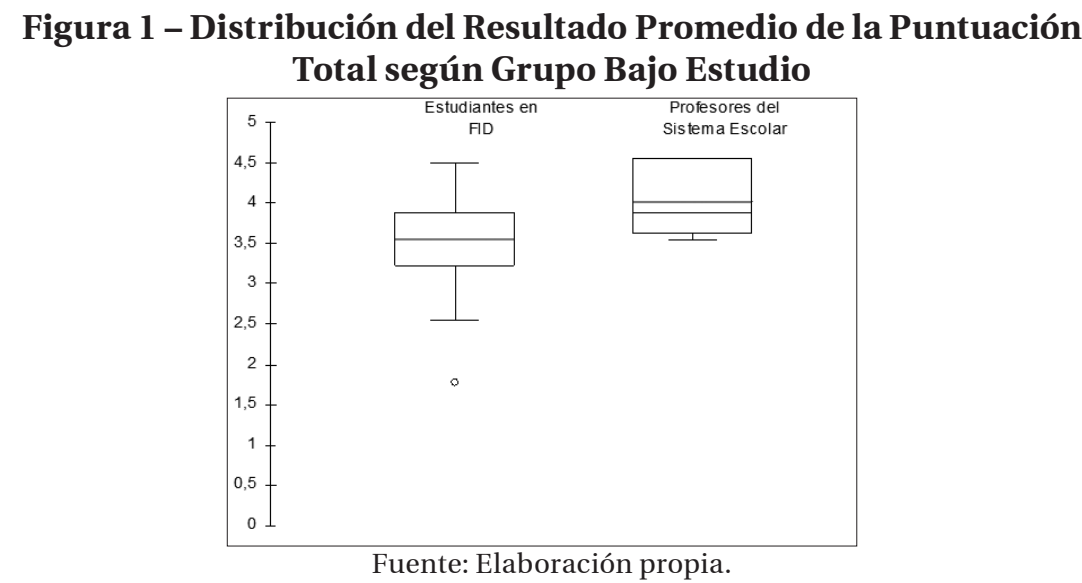

Educação \& Realidade, Porto Alegre, v. 42, n. 4, p. 1459-1477, oct./dic. 2017. 
Alfabetizacion Estadistica en Profesores de Distintos Niveles Formativos

Observando que los profesores del sistema escolar presentan una menor variabilidad en sus respuestas en relación a los estudiantes en FID cuya distribución es relativamente simétrica (Coeficiente de Asimetría aproximadamente cero). Lo cual puede ser explicado en que los profesores del sistema escolar han asimilado en sus actividades escolares que la estadística permite modelar situaciones de la vida diaria permitiendo mostrar una realidad social más allá de los procedimientos algorítmicos, habilidades declaradas en los estándares fijados por el Ministerio de Educación chileno.

\section{Habilidades de Alfabetización Estadística de los Estudiantes FID y Profesores en Ejercicio del Sistema Escolar}

Uno de los conceptos ampliamente utilizados en estadística es el de muestra y su representatividad aludiendo a tipos de muestreos (muestreo aleatorio simple, muestreo sistemático, estratificado y por conglomerado), puesto que ellos juegan un rol fundamental en la inferencia estadística. A nivel del sistema escolar chileno son conceptos que se desarrollan en el currículo escolar a partir de octavo año básico. Cabe mencionar que en ese nivel de escolaridad los alumnos tienen una edad promedio de 14 años y se encuentran en el estadio de operaciones formales.

En coherencia con lo anterior en el instrumento aplicado se introdujeron reactivos tendientes evaluar las habilidades de los estudiantes en FID y profesores en ejercicio del sistema escolar sobre conceptos básicos de muestreo. En la siguiente tabla (Tabla 6) se muestran los porcentajes de logro de los grupos bajo estudio.

Tabla 6 - Porcentaje Promedio de logro entre Estudiantes en Formación Inicial Docente y Profesores del Sistema Escolar

\begin{tabular}{|l|c|c|c|}
\hline & $\begin{array}{c}\text { Estudiantes en } \\
\text { Formación Inicial } \\
\text { Docente }\end{array}$ & $\begin{array}{c}\text { Profesores del } \\
\text { Sistema Escolar }\end{array}$ & p-Valor \\
\hline $\begin{array}{l}\text { Identificar la representati- } \\
\text { vidad de la muestra en una } \\
\text { población objeto de estudio. }\end{array}$ & 73,3 & 60,0 & 0,40 \\
\hline $\begin{array}{l}\text { Inferencia hacia una poblaci- } \\
\text { ón objeto de estudio en base a } \\
\text { información muestral. }\end{array}$ & 76,2 & 100,0 & 0,00 \\
\hline
\end{tabular}

Fuente: Elaboración propia.

El porcentaje promedio más descendido en relación a la habilidad de identificar muestra y su representatividad se observa en el grupo de profesores en ejercicio del sistema escolar alcanzado un $60,0 \%$ y los estudiantes en FID obtienen en promedio un 73,3\% frente al mismo reactivo. Estos resultados porcentuales pueden ser explicados debido a que la formación estadística recibida por los sujetos bajo estudio en general es de carácter algorítmica (Estrella; Olfos; Mena-Lorca, 2015), dichas diferencias numéricas porcentuales entre los grupos no son estadísticamente significativas (valor $\mathrm{p}=0,40$ ).

1470 Educação \& Realidade, Porto Alegre, v. 42, n. 4, p. 1459-1477, oct./dic. 2017. 
Cabe señalar que muestra y su representatividad son aspectos de interés en estadística, permitiendo inferir cuánto se parece a la población de la cual se obtuvo. En este sentido a fin de profundizar en los conceptos estadísticos del grupo de estudio, se realizó un análisis de los argumentos entregados por los encuestados, para justificar la opción de respuesta. En el primer caso solamente un 6,5\% argumenta de manera correcta, explicitando que la muestra considerada en el enunciado de la situación problemática no es representativa de la población objeto de estudio aun cuando esto se enuncia de manera explícita en el enunciado del reactivo. Un 4,3\% declara que la muestra no es de carácter aleatoria, no obstante en el enunciado nada se dice al respecto. Así mismo un 4,3\% alude al concepto de población de lo cual se infiere una confusión entre los conceptos de población y muestra. Finalmente un $28,3 \%$ de los encuestados entregan argumentaciones ajenas al contexto del enunciado.

Otra característica del proceso inferencial es tomar decisiones a nivel poblacional en base a información muestral. En este sentido un $17,4 \%$ de los encuestados relatan que los estadígrafos solamente permiten describir información de la muestra sin inferir a nivel poblacional. Un 2,2\% declaran en su argumentación que el resumen numérico entregado no corresponde a un estadígrafo. Por otro, lado hay un $26,1 \%$ de los encuestados que justifica en base a contextos ajenos al enunciado entregado.

Al estudiar la correlación de los resultados promedios en relación al concepto de representatividad de una muestra con la población objeto de estudio, se presenta una correlación positiva estadísticamente significativa con el componente cognitivo es fundamental en la formación básica del futuro ciudadano $(\mathrm{r}=0,31$, valor $\mathrm{p}=0,00)$. En cambio con el componente comportamental A menudo explico a mis compañeros problemas de estadística que me han entendido se correlaciona de manera inversa $(\mathrm{r}=-0,21$, valor $\mathrm{p}=0,03)$.

Por otro lado cuando se observan los porcentajes promedios de logro en relación con la habilidad de inferir en base a información muestral existen diferencias significativas al 5\% entre ambos grupos. El $100,0 \%$ de los profesores en ejercicio del sistema escolar responden correctamente este reactivo, mientras que este porcentaje disminuye a un $76,2 \%$ en el caso de los estudiantes en formación inicial docente.

Otro de los conceptos básicos en estadística que deben conocer los estudiantes en FID y profesores en ejercicio del sistema escolar es lo relativo al concepto de estadístico (o estadígrafo) cuyo objetivo es describir el comportamiento numérico de información muestral. Es un objeto estadístico que tiene la característica de ser variable permitiendo inferir comportamientos a nivel poblacional. Adicionalmente los sujetos deben manejar el concepto de parámetro el cual permite resumir información a nivel poblacional. Se trata de un objeto estadístico caracterizado por ser constante y casi siempre es desconocido. Se suele simbolizar mediante letras griegas. 
Alfabetizacion Estadistica en Profesores de Distintos Niveles Formativos

En la siguiente tabla (Tabla 7) se muestran los porcentajes de logro de los estudiantes en FID y profesores en ejercicio del sistema escolar al momento de hacer inferencias en base a la información entregada por un estadígrafo. Se observa que ambos grupos tienen porcentajes de logro similares del orden del $28,7 \%$ y $26,7 \%$ respectivamente, lo que equivale a resultados descendidos. Sin embargo, cuando deben interpretar un estadígrafo y decidir sobre su representatividad, los profesores del sistema escolar presentan un porcentaje de logro promedio de $46,7 \%$, valor superior al obtenido por los estudiantes en FID (29,3\%). No obstante, esta diferencia no llegar a ser estadísticamente significativa.

Tabla 7 - Porcentaje Promedio de Logro entre Estudiantes en Formación Inicial Docente y Profesores del Sistema Escolar

\begin{tabular}{|l|c|c|c|}
\hline & $\begin{array}{c}\text { Estudiantes } \\
\text { en Formación } \\
\text { Inicial Docente }\end{array}$ & $\begin{array}{c}\text { Profesores del } \\
\text { Sistema Escolar }\end{array}$ & p-Valor \\
\hline $\begin{array}{l}\text { Inferencia en base a informa- } \\
\text { ción obtenida mediante un } \\
\text { estadístico. }\end{array}$ & 28,7 & 26,7 & 0,90 \\
\hline $\begin{array}{l}\text { Interpretación de un estadí- } \\
\text { grafo y su representatividad. }\end{array}$ & 29,3 & 46,7 & 0,20 \\
\hline
\end{tabular}

Fuente: Elaboración propia.

A continuación se comentan los resultados de los sujetos bajo estudio en un reactivo elaborado para evaluar habilidades inferenciales. El enunciado presenta la situación hipotética de estudiantes de una facultad que asisten a un centro de fotocopiado en una determinada hora del día, con el objetivo de realizar inferencias de carácter intuitiva acerca de la población objeto de estudio. Cabe mencionar que solamente un $8,9 \%$ de los encuestados afirma que la muestra es representativa, situación que es coherente con el enunciado del reactivo. Por otro lado un $23,9 \%$ de los sujetos alude a que la muestra no es representativa y el $67,2 \%$ entrega razones poco plausibles en relación a conceptos estadísticos.

En un segundo reactivo, se solicita interpretar un estadígrafo y estudiar su representatividad, en el contexto de una encuesta de opinión sobre la segunda vuelta de la candidatura presidencial en Chile efectuada el año 2013, en cuyo proceso el candidato ganador obtuvo el $62,2 \%$. El 23,9\% de los encuestados argumentan que dicho porcentaje representa los votos válidamente emitidos y un 10,9\% declara que se trata del porcentaje de votantes, utilizando en su explicación frases entregadas literalmente en el enunciado de reactivo.

Existen correlaciones estadísticamente significativas entre la idea de inferencia en base a información obtenida mediante un estadístico y el componente pedagógico cognitivo es fundamental en la formación básica del futuro ciudadano directa $(\mathrm{r}=0,26$, valor $\mathrm{p}=0,009)$, y con el componente comportamental A menudo explico a mis compañeros problemas de estadística que no han aprendido inversa $(\mathrm{r}=-0.27$, valor $\mathrm{p}$ $=0,02$ ). Por otro lado la interpretación de un estadígrafo y su represen-

1472 Educação \& Realidade, Porto Alegre, v. 42, n. 4, p. 1459-1477, oct./dic. 2017. 
tatividad se correlaciona de manera directa con el componente afectivo encuentro interesante el mundo de la estadística.

La lectura e interpretación de tablas de doble entrada donde participan frecuencias relativas (o porcentuales) es una de las habilidades a desarrollar por los futuros estudiantes. En la siguiente tabla se muestra los porcentajes de logro de los grupos bajo estudio, donde los profesores en ejercicio del sistema escolar presentan un porcentaje más descendido $(33,3 \%)$ en relación a los estudiantes en FID $(64,4 \%)$ en la descodificación de información presentada en una tabla de contingencia, existiendo diferencias estadísticamente significativa al 5\%,. En relación a la lectura de gráficos de barras agrupadas (información bidimensional) el $58,1 \%$ de los estudiantes en FID logran descodificar la información presente en dicha representación gráfica, porcentaje que disminuye a un $46,7 \%$ en los profesores en ejercicio del sistema escolar, cuya diferencia promedio porcentual no llega a ser estadísticamente significativa (ver Tabla 8).

Tabla 8 - Porcentaje Promedio de Logro entre Estudiantes en Formación Inicial Docente y Profesores del Sistema Escolar en Relación a la Decodificación de Información en Resumida en Tablas de Contingencia y Representaciones Gráficas

\begin{tabular}{|l|c|c|c|}
\hline & $\begin{array}{c}\text { Estudiantes } \\
\text { en Formación } \\
\text { Inicial Docente }\end{array}$ & $\begin{array}{c}\text { Profesores del } \\
\text { Sistema Escolar }\end{array}$ & p-Valor \\
\hline $\begin{array}{l}\text { Lectura de frecuencias relativas } \\
\text { porcentuales en tablas de doble } \\
\text { entrada. }\end{array}$ & 64,4 & 33,3 & 0,02 \\
\hline $\begin{array}{l}\text { Lectura de representaciones } \\
\text { gráficas bidimensionales. }\end{array}$ & 58,1 & 46,7 & 0,20 \\
\hline
\end{tabular}

Fuente: Elaboración propia.

El 50,0\% de los sujetos bajo estudio argumenta la elección de la alternativa se basó fundamentalmente en la lectura de la primera fila de la tabla de doble entrada, un $15,2 \%$ consideró una lectura bidimensional (intersección de la primera fila y segunda columna) y un 6,5\% observó la redacción de las distintas alternativas para la toma de decisiones.

La decodificación de representaciones gráficas es una de las habilidades que deben desarrollar tanto futuros estudiantes en FID como profesores del sistema escolar. Por lo anterior se les solicita efectuar un análisis de las tendencias presentes en un gráfico de barras agrupadas que resume la relación del nivel educativo según género en dos años diferentes. El 54,3\% de los sujetos bajo estudio argumenta correctamente su elección en base a la altura de las barras, las cuales evidencian que los valores porcentuales son muy similares y el $4,3 \%$ de los encuestados argumenta que hay más hombres que mujeres, no obstante las alternativas dan cuenta de valores aproximados.

La lectura de frecuencias relativas porcentuales en tablas de doble entrada se correlaciona significativamente con los componentes 
Alfabetizacion Estadistica en Profesores de Distintos Niveles Formativos

cognitivo es fundamental en la formación básica del futuro ciudadano $(\mathrm{r}=0,28$, valor $\mathrm{p}=0,006)$ y con el componente comportamental A menudo explico a mis compañeros problemas de estadística que no han entendido $(\mathrm{r}=0,23$, valor $\mathrm{p}=0,02)$.

Por otro lado en la descodificación de información resumida en representaciones de gráficas bidimensionales se correlaciona con los componentes pedagógicos (Afectivos, cognitivos y comportamentales), en particular en forma negativa con el componente En la escuela no habría que enseñar estadística $(\mathrm{r}=-021$, valor $\mathrm{p}=0,03)$, los problemas de estadística me resulta fáciles ( $\mathrm{r}=-0,27$, valor $\mathrm{p}=0,01)$, La estadística es fácil $(\mathrm{r}=-0,26$, valor $\mathrm{p}=0,01)$ y Si pudiera eliminar alguna materia sería la estadística $(\mathrm{r}=-023$, valor $\mathrm{p}=0,02)$.

\section{Conclusiones}

El estudio realizado tenía el propósito de indagar sobre el nivel de alfabetización y la relación con la Escala de Actitudes hacia la Estadística introducida por Estrada (2011) que evidencian estudiantes en Formación Inicial Docente y profesores en ejercicio del sistema escolar.

Los resultados permiten concluir que en general, los sujetos presentan diferencias significativas en relación a las actitudes hacia la estadística, evidenciando que los profesores en ejercicio del sistema escolar entregan resultados promedios más altos en los componentes encuentro interesante el mundo de la estadística, los problemas de estadística me resulta fáciles y En clase de estadística nunca entiendo de qué están hablando que los estudiantes en Formación Inicial Docente. El análisis de varianza factorial arrojó solamente diferencias significativas entre estudiantes en FID y profesores en ejercicio del sistema escolar, situación que no se da en el factor género (femenino, masculino) en la puntuación total de los componentes de actitudes hacia la estadística, considerados en el presente artículo.

En relación a las habilidades de alfabetización estadística se concluye que la inferencia e interpretación de estadígrafos en situaciones contextualizadas presentan los porcentajes de logro más descendidos tanto en los estudiantes en FID como en profesores en ejercicio del sistema escolar. Situación similar se observa en la descodificación de información resumida en tablas de doble entrada (o de contingencia) y representaciones gráficas bidimensionales (gráfico de barras agrupadas), lo cual es coherente con los hallazgos de Alveal y Rubilar (2012).

Desde el punto de vista de la argumentación entregada por los grupos encuestados es posible afirmar que los sujetos esgrimen argumentos poco plausibles como así también incoherentes con los saberes estadísticos tales como muestra, representatividad, estadígrafos y parámetros y su relación con la inferencia estadística. En general este hallazgo llama la atención, teniendo presente que la argumentación es una de las habilidades que los profesores deben desarrollar en su trayectoria formativa en coherencia con los estándares fijados por el Ministerio de Educación chileno.

1474 Educação \& Realidade, Porto Alegre, v. 42, n. 4, p. 1459-1477, oct./dic. 2017. 
Por otro lado, se presentan correlaciones estadísticamente significativas entre los porcentajes de logro del instrumento de alfabetización y los resultados del componente cognitivo es fundamental en la formación básica del futuro ciudadano, evidenciando que los estudiantes en FID y profesores del sistema escolar tienen conciencia que la estadística es fundamental en la formación de los ciudadanos, puesto que juega un rol fundamental en la alfabetización estadística, siendo coherente con los hallazgos de Tauber, Albrecht y Bertorello (2011).

Finalmente el presente estudio, proporciona evidencias sobre cómo los estudiantes en Formación Inicial Docente y profesores en ejercicio del sistema escolar visualizan la estadística, y también aporta información de las habilidades de conceptos básicos relacionados con la alfabetización estadística. Estos resultados podrían favorecer la reflexión en torno a núcleos temáticos para profundizar en el desarrollo profesional de los futuros profesores, y de los que actualmente se encuentran insertos en el sistema escolar chileno, si lo anterior se plasmara en el currículo de Formación Inicial Docente y las políticas de capacitación y actualización de los profesores en ejercicio, a objeto de mejorar sus niveles de desarrollo en dichas habilidades. Al mismo tiempo resulta de interés la construcción de un instrumento que aborde varias dimensiones las cuales permitan evaluar los niveles de alfabetización estadística atendiendo a los lineamientos ministeriales ${ }^{2}$.

Recibido en 29 de Febrero de 2016 Aprobado en 13 de Mayo de 2016

\section{Notas}

1 En Chile la tipificación de los establecimientos educaciones del sistema escolar es Municipal (Establecimientos públicos de propiedad y financiamiento principalmente estatal), Particular Subvencionado (Establecimiento de propiedad y administración privada que reciben financiamiento estatal en subvención por alumnos matriculados) y Particular Pagado (establecimientos privados cuya propiedad, administración y financiamiento corresponde a particulares). Disponible en: <http://www.oei.es/quipu/chile/CHIL04.PDF>. Acceso en 15 jul. 2015.

2 El autor agradece a los evaluadores del artículo, por sus valiosos comentarios y sugerencias que han permitido mejorar la comunicación de los resultados del estudio.

\section{Referencias}

ALVEAL, Francisco Rodríguez; FUENTES, Ana Carolina Maldonado; RUBILAR, Pedro Rodrigo Sandoval. Comprensión de las Medidas de Tendencia Central: un estudio comparativo en estudiantes de pedagogía en matemática en dos instituciones formadoras chilenas. Avaliação: Revista de Avaliação da Educação Superior, Sorocaba, v. 21, n. 3, p. 929-952, nov. 2016.

ALVEAL, Francisco Rodríguez; RUBILAR, Pedro Rodrigo Sandoval. Habilidades de Codificación y Descodificación de Tablas y Gráficos Estadísticos: un estudio 
Alfabetizacion Estadistica en Profesores de Distintos Niveles Formativos

comparativo en profesores y alumnos de pedagogía en enseñanza básica. Revista da Avaliação da Educação Superior, Campinas, v. 17, n. 1, p. 207-235, mar. 2012.

AZORÍN, Francisco; SANCHEZ, José Luis. Métodos y Aplicaciones del Muestreo. Madrid: Alianza, 1986.

BATANERO, Carmen. Los Retos de la Cultura Estadística: conferencia inaugural. In: JORNADAS INTERAMERICANAS DE ENSEÑANZA DE LA ESTADÍSTICA, 2002, Buenos Aires. Anais... Buenos Aires: 2002. P. 1-11.

CASTELLS, Manuel. Globalización, Sociedad y Política en la Era de la Información. Revista Análisis Político, Bogotá, v. 1, n. 37, p. 2-17, mayo/ago. 1999. Disponible en: <http://biblioteca.clacso.edu.ar/ar/libros/colombia/assets/own/ analisis37.pdf>. Acceso en: 8 Jun. 2015

CHILE. Resultados TIMSS 2011: estudio internacional de tendencias en matemática y ciencias. Santiago de Chile: Agencia de Calidad de la Educación; IEA, 2011. Disponible en: <http://www.agenciaeducacion.cl/wp-content/ uploads/2013/02/resultados-timss-18-dic-2012.pdf>. Aceso en: 27 Jul. 2015.

CHILE. Bases Curriculares 2012: matemática. Santiago: Ministerio de Educación, 2012. Disponible en: <http://www.curriculumenlineamineduc.cl/605/articles-21321_programa.pdf>. Acceso en: 28 Jun. 2015.

COUTINHO, Cileda de Queiroz e Silva; ALMOULOUD, Saddo Ag; SILVA, Maria José Ferreira da. O Desenvolvimento do Letramento Estatístico a partir do Uso do Geogebra: um estudo com professores de matemática. Revemat: Revista Eletrônica de Educação Matemática, Florianópolis, v. 7, n. 2, p. 246-265, jun. 2012.

CRAVERO, Mariela; REDONDO, Yanina; SANTELLAN, Silvana. Competencias en Educación Estadística: de una alfabetización estadística hacia una alfabetización científica. In: CONFERENCIA INTERAMERICANA DE EDUCACIÓN MATEMÁTICA, 13., 2011, Recife. Anais... Recife: 2011. P. 26-36. Disponible en: $<$ http://www.gente.eti.br/lematec/CDS/XIIICIAEM/artigos>. Acceso en: 15 Ago. 2015.

ESTRADA, Assumpta. Instrumentos de Medición de Actitudes hacia la Estadística: la escala EAEE para profesores: investigación en educación matemática. In: COMUNICACIONES DE LOS GRUPOS DE INVESTIGACIÓN DE LA SEIEM, 2011, Lleida. Anais... Lleida: 2011. P. 233-251.

ESTRELLA, Soledad; OLFOS, Raimundo; MENA-LORCA, Arturo. Pedagogical Knowledge of Statistics Content Among Primary School Teachers. Educação e Pesquisa, Sao Paulo, v. 41, n. 2, p.477-493, jun. 2015.

GAL, Iddo. Statistical Literacy Meanings, Components, Responsibilities. In: BEN-ZVI, Dani; GARFIELD, Joan (Ed.). The Challenge of Developing Statistical Literacy, Reasoning and Thinking. Netherlands: Springer, 2004. P. 47-78. Disponible en: <http://link.springer.com/chapter/10.1007/1-4020-2278-6_3>. Acceso en: 25 Mayo 2015.

GAL, Iddo; GINSBURG, Lynda; SCHAU, Candace. Monitoring Attitudes and Beliefs in Statistics Education. In: GALL, Iddo; GARFIELD, Joan. The Assessment Challenge in Statistics Education. Amsterdam: Ios Press, 1997. P. 37-51. Disponible en: <https://www.stat.auckland.ac.nz/ iase/publications/assessbkref>. Acceso en: 25 Mayo 2015.

GARFIELD, Joan; DELMAS, Robert; CHANCE, Beth. The Web-Based ARTIST: assessment resource tools for improving statistical thinking. In: ASSESSMENT OF STATISTICAL REASONING TO ENHANCE EDUCATIONAL QUALITY, AT THE AN-

1476 Educação \& Realidade, Porto Alegre, v. 42, n. 4, p. 1459-1477, oct./dic. 2017. 
NUAL MEETING OF THE AMERICAN EDUCATIONAL RESEARCH ASSOCIATION (AERA), 2003, Chicago. Anais... Chicago: 2003. P. 1-22. Disponible en: <https:// apps3.cehd.umn.edu/artist/articles/AERA_2003.pdf>. Acceso en: 10 Jul. 2015.

KATAOKA, Veronica Yumi et al. Ambiente Virtual de Apoio ao Letramento Estatístico-AVALE. In: ENCONTRO NACIONAL DE EDUCAÇÃO MATEMÁTICA: EDUCAÇÃO MATEMÁTICA, CULTURA E DIVERSIDADE, 10., 2010, Salvador. Anais... Salvador: 2010. P. 1-6.

MAFOKOZI, Joseph. Nivel de Alfabetización Estadística del Alumnado Universitario de Letras: el caso de la facultad de educación de la universidad complutense de Madrid. Revista Complutense de Educación, Madrid, v. 22, n. 1, p. 95125, Mayo 2011.

NIETO, Lorenzo J. Blanco; LOPEZ, Manuel Barrantes. Concepciones de los Estudiantes para Maestro en España sobre la Geometría Escolar y su Enseñanza y Aprendizaje. Revista Latinoamericana de Investigación En Matemática Educativa, Ciudad de Mexico, v. 6, n. 2, p. 107-132, 2003.

PINO, Guido del; ESTRELLA, Soledad. Educación Estadística: relaciones con la matemática. Pensamiento Educativo: Revista de Investigación Educacional Latinoamericana, Santiago, v. 49, n. 1, p. 53-64, Mayo 2012.

ROCA, María Asunción Estrada. Análisis de las Actitudes y Conocimientos Estadísticos Elementales en la Formación del Profesorado. 2002. 117 f. Tesis (Doctoral) - Departament de Didàctica de la Matemàtica i de les Ciències Experimentals, Universidad Autónoma de Barcelona, Barcelona, 2002.

SERRADÓ, Ana. El Proyecto Internacional de Alfabetización Estadística. Números Revista de Didáctica de Las Matemáticas, Tenerife, v. 83, p. 19-33, Jun. 2013.

TAUBER, Liliana; ALBRECHT, Gisela; BERTORELLO, Noelia. Estudio sobre Actitudes y Elementos de Significado Utilizados por Profesores de Matemática en la Resolución de Tareas Estadísticas. In: CONFERENCIA INTERAMERICANA DE EDUCACIÓN MATEMÁTICA, 13., 2011, Recife. Anais... Recife: 2011. P. 12-16. Disponible en: <http://www.gente.eti.br/lematec/CDS/XIIICIAEM/artigos>. Acceso en: 12 Mar. 2015.

UNITED NATIONS EDUCATIONAL, SCIENTIFIC AND CULTURAL ORGANIZATION. Día Internacional de la Alfabetización de 2015. Paris: Unesco, 2015. Disponible en: <http://www.unesco.org/new/es/unesco/events/prizes-and-celebrations/celebrations/international-days/literacy-day/>. Acceso en: 15 Jul. 2015.

WALLMAN, Katherine. Enhancing Statistical Literacy: enriching our society. Journal of the American Statistical Association, Washington, p. 1-8. mar. 1993.

WATSON, Joan. Assessing Statistical Thinking. In: GAL, Iddo; GARFIELD, Joan (Ed.). The Assessment Challenge in Statistics Education. Netherlands: IOS Press, 1997. P. 107-121.

Francisco Enrique Rodriguez-Alveal és profesor de Educacion En Matematica y Magister en Estadistica actualmente trabajo en el Departamento Ciencias de la Educacion de la Universidad del Bío-Bío de Chillán. Lineas de interes son Formacion Inicial Docente, Evaluación, Didactica de la Estadistica y Teoria de Distribuciones.

E-mail:frodriguez@ubiobio.cl 